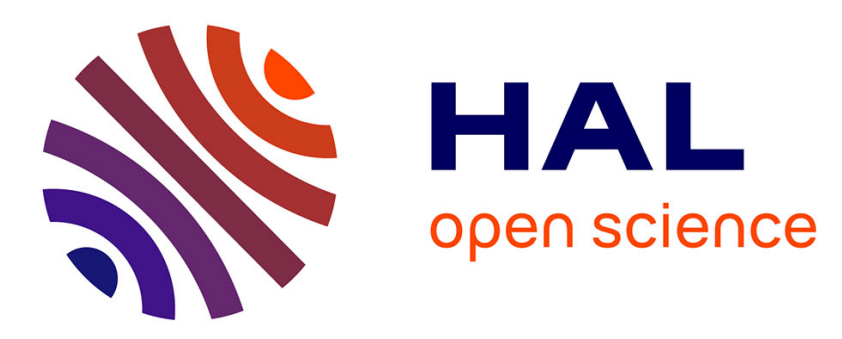

\title{
FuRIA: A Novel Feature Extraction Algorithm for Brain-Computer Interfaces using Inverse Models and Fuzzy Regions of Interest
}

Fabien Lotte, Anatole Lécuyer, Bruno Arnaldi

\section{- To cite this version:}

Fabien Lotte, Anatole Lécuyer, Bruno Arnaldi. FuRIA: A Novel Feature Extraction Algorithm for Brain-Computer Interfaces using Inverse Models and Fuzzy Regions of Interest. 3rd IEEE-EMBS international conference on neural engineering, May 2007, Hawaii - Kohala Coast, United States. inria-00134961

\section{HAL Id: inria-00134961 \\ https://hal.inria.fr/inria-00134961}

Submitted on 6 Mar 2007

HAL is a multi-disciplinary open access archive for the deposit and dissemination of scientific research documents, whether they are published or not. The documents may come from teaching and research institutions in France or abroad, or from public or private research centers.
L'archive ouverte pluridisciplinaire HAL, est destinée au dépôt et à la diffusion de documents scientifiques de niveau recherche, publiés ou non, émanant des établissements d'enseignement et de recherche français ou étrangers, des laboratoires publics ou privés. 


\title{
FuRIA: A Novel Feature Extraction Algorithm for Brain-Computer Interfaces using Inverse Models and Fuzzy Regions of Interest
}

\author{
Fabien Lotte, Anatole Lécuyer, Bruno Arnaldi
}

\begin{abstract}
In this paper, we propose a new feature extraction algorithm for Brain-Computer Interfaces (BCIs). This algorithm is based on inverse models and uses the novel concept of fuzzy Region Of Interest (ROI). It can automatically identify the relevant ROIs and their reactive frequency bands. The activity in these ROIs can be used as features for any classifier. A first evaluation of the algorithm, using a Support Vector Machine (SVM) as classifier, is reported on data set IV from BCI competition 2003. Results are promising as we reached an accuracy on the test set ranging from $85 \%$ to $86 \%$ whereas the winner of the competition on this data set reached $84 \%$.
\end{abstract}

\section{INTRODUCTION}

Brain-Computer Interfaces (BCIs) are communication systems that enable to send commands only by means of brain activity [1]. ElectroEncephaloGraphy (EEG) is the most common way of measuring this activity for a BCI system. Consequently, identifying the brain activity through EEG has become a major challenge in the design of efficient BCIs. Two key points are involved in this identification, namely, feature extraction and classification [2]. Feature extraction aims at describing the EEG stream by a few relevant values called features while classification aims at automatically assigning a class to these features. In this paper we address the problem of feature extraction for which it is admitted that the exploration of new algorithms is necessary [3].

During the last 3 years, inverse models have shown to be promising feature extraction algorithms [4] [5] [6] [7]. Such models are able to compute the activity in the whole brain volume, only using EEG and a head model that generally represents the brain as a set of voxels (volume elements). The activity thus calculated in some relevant brain regions or voxels has been used as efficient features for BCI systems.

Despite recurrent good results, some limitations remain. Actually, it appears difficult to conciliate both generecity, i.e., the ability to deal with any kind of mental task, and generation of few features. On one hand, automatic and generic methods generate a very high number of features, due to the consideration of the activity of single voxels [5]. Actually, these voxels can be dependant and as such should be gathered in brain regions. On the other hand, methods generating few features were proposed but are not generic anymore as they require a priori knowledge

This work was supported by the French National Research Agency and the National Network for Software Technologies within the Open-ViBE project and grant ANR05RNTL01601.

F. Lotte, A. Lécuyer and B. Arnaldi are with IRISA / INRIA Rennes, Campus universitaire de Beaulieu, Avenue du Général Leclerc, 35042 RENNES Cedex, FRANCE E-mail: fflotte, alecuyer, arnaldi\}@irisa.fr and are only suitable for motor imagery [4] [6]. Finally, a recently proposed method was both generic and generator of few features due to the gathering of dependant voxels into Regions Of Interest (ROIs). However, this method suffered from not being fully automatic and being limited to the use of two ROIs which spatial extension was hard to define [7].

This paper aims at addressing those limitations. Indeed, we propose a generic feature extraction algorithm that can automatically identify any number of relevant ROIs and can properly define their spatial extension thanks to the novel concept of fuzzy ROI. We called this algorithm FuRIA, which stands for Fuzzy Region of Interest Activity.

\section{THE FURIA ALgORITHM}

FuRIA is a trainable feature extraction algorithm. Indeed, it can automatically identify what are the relevant ROIs for mental task discrimination and in which frequency bands they react. Describing the brain activity using FuRIA consists in computing the activity in those ROIs, in their corresponding frequency bands. This Section proposes an overview of FuRIA and describes the different steps that compose it.

\section{A. Overview of the algorithm}

In order to use FuRIA, an initial training phase is required. This phase is performed offline and is divided into five steps which will be described more deeply in the next Sections:

1) Statistical analysis: A statistical analysis comparing the different classes (mental tasks) is performed for each frequency and each voxel. The goal is to identify the voxels which activity for a given frequency can discriminate the classes.

2) Clustering: A clustering algorithm is performed in order to gather voxels, which activity is statistically discriminative, into different ROIs.

3) Fusion: ROIs found at similar spatial locations, in consecutive frequencies, are fusionned and identified as reactive in the frequency band resulting from the concatenation of these frequencies.

4) Adaptation: For each ROI obtained during fusion, another statistical analysis is performed in order to remove voxels that are not significant anymore in the reactive frequency band identified at the fusion step.

5) Fuzzification: Each ROI is "fuzzified", i.e., turned as a fuzzy ROI (cf. Section II-F) in order to properly define its spatial extension.

Once the training phase is achieved, feature extraction consists in filtering the EEG signals in the reactive frequency band of each fuzzy ROI found and then using a given inverse 
model to compute its activity. These activity values are then concatenated into a single feature vector that can be used with any classifier. The following sections detail these five training steps.

\section{B. Statistical Analysis}

This step consists in comparing, using a statistical test, the mean value of each voxel activity, possibly averaged over a chosen time window, between the different classes. This statistical analysis is performed separately for each one of the $N_{f}$ considered frequencies. Once this analysis is done, we obtain $N_{f}$ statistical maps, each one revealing which voxels have an activity that is statistically different between the classes for the corresponding frequency. These voxels, that we denote as significant voxels, are potential candidates as members of the relevant ROIs which activity will eventually be used for discrimination.

To deal with a binary BCI, the familly of t-tests can be used as a statistical analysis whereas to deal with a multiclass BCI, the familly of ANOVA tests can be used instead.

\section{Clustering}

For each statistical map computed at the previous step, a clustering algorithm is performed in order to aggregate significant voxels into different ROIs. In order to do this clustering, a 4-dimensionnal vector $[x, y, z, s]$ is associated to each significant voxel. The coordinates $x, y, z$ are the $3 \mathrm{D}$ coordinates of the voxel in the chosen head model, and $s$ is the voxel statistic computed at the previous step. Thus, we work in the joint space-range domain in order to find significant voxels that are spatially gathered, and that share common statistic values. This last point is essentially done to prevent the gathering of a voxel with a positive statistic together with a voxel with a negative statistic in the same ROI. Once the clustering performed, all the voxels which corresponding vectors belong to the same cluster are aggregated into the same ROI. Optionnaly, very small ROIs, containing only one or two voxels for instance, could be removed. This step done, a set of relevant ROIs have been identified for each frequency.

The clustering algorithm used must be able to automatically identify any number of clusters and should not have too strong assumptions about these cluster shapes.

\section{Fusion}

As we have found what the relevant ROIs were for each frequency, we need to define which ROIs are relevant in which frequency bands. To do so, we follow this procedure:

1) Associate each ROI $\Omega$ found previously with the frequency $f$ at which it was found;

2) Among the whole set of couples $(\Omega ; f)$, select two couples $\left(\Omega_{1} ; f_{1}\right)$ and $\left(\Omega_{2} ; f_{2}\right)$, such that the overlap between $\Omega_{1}$ and $\Omega_{2}$ is high, and the frequency bands $f_{1}$ and $f_{2}$ are overlapped or are consecutive. We consider an overlap is high if $\operatorname{card}\left(\Omega_{1} \cap \Omega_{2}\right)>$ $0.5 * \min \left(\operatorname{card}\left(\Omega_{1}\right), \operatorname{card}\left(\Omega_{2}\right)\right)$ with $\operatorname{card}(\Omega)$ being the number of voxels in $\Omega$.
3) replace the couples $\left(\Omega_{1} ; f_{1}\right)$ and $\left(\Omega_{2} ; f_{2}\right)$ by the single couple $\left(\Omega_{1} \cup \Omega_{2} ; f_{1} \cup f_{2}\right)$. This means we choose the Union as the way of fusionning ROIs;

4) Return to point 2, until no more ROIs can be fusionned.

\section{E. Adaptation}

Due to the use of the Union as the fusion operator, a ROI $\Omega$ identified as reactive in the frequency band $\left[f_{a} ; f_{b}\right]$ may contain voxels that were significant in a frequency between $f_{a}$ and $f_{b}$ but that are not significant anymore in $\left[f_{a} ; f_{b}\right]$. Therefore, for each ROI found at the previous step, we perform another statistical analysis for the ROI voxels, in its associated reactive frequency band in order to remove nonsignificant voxels. Moreover, the statistics found at this adaptation step will be used for the next training step.

\section{F. Fuzzification and the concept of fuzzy ROI}

In the ROIs we have defined so far, we can notice that all the voxels do not have the same statistical significance. However, these voxels are all significant, which means they still carry useful information. Moreover, electrophysiologically, the brain regions related to specific brain functions are not well defined for a specific user: their boundaries are naturally "fuzzy". Consequently, identifying which voxels should be kept in the ROI is a major problem.

We believe that any voxel carrying information should be in the ROI, but that those with less information should be "less" in the ROI. Therefore, we propose the concept of fuzzy ROI which is based on the concept of fuzzy sets developed by Zadeh [8]. A classical ROI $\Omega_{c}$ is defined by the set of voxels it contains. The activity $\gamma_{\Omega_{c}}$ inside this ROI $\Omega_{c}$ is classically computed by:

$$
\gamma_{\Omega_{c}}=\sum_{v \in R O I} \gamma_{v}
$$

where $\gamma_{v}$ is the activity of the voxel $v$.

A fuzzy ROI $\Omega_{f}$ is not defined by a set of voxels anymore but by a fuzzy membership function $\mu$. This function provides the degree of membership, in $[0 ; 1]$, of any existing voxel to the fuzzy ROI $\Omega_{f}$. This leads to fuzzy ROIs which boundaries are not well defined and therefore fuzzy. The activity of a fuzzy ROI is computed as follows:

$$
\gamma_{\Omega_{f}}=\sum_{v=1}^{N_{v}} \mu(v) \gamma_{v}
$$

with $N_{v}$ being the number of voxels in the whole head model. This formalism makes it possible to weigh each voxel according to its relative contribution in the ROI, and as such, use efficiently the whole of the available information.

To achieve FuRIA training, we fuzzify all the ROIs $\Omega$ by associating to each one of them a fuzzy membership function $\mu_{\Omega}$ which uses the statistics computed at the adaptation step. The shape of this function $\mu_{\Omega}$ depends on the inverse model and the statiscal test used. In section III we propose a suitable fuzzy membership function for the 
algorithms we used.

Once the automatic training of FuRIA is achieved, a set of fuzzy ROIs relevant for discrimination has been identified as well as their corresponding reactive frequency bands. The activity in these fuzzy ROIs and in these frequency bands, possibly averaged over a time window, can then be used as features for any classifier. This is highlighted in Section IV which is devoted to a first evaluation of FuRIA.

\section{IMPLEMENTATION OF THE ALGORITHM}

Due its decomposition into different steps, FuRIA is a generic algorithm that can be used with different implementations for each of these steps. We report here the inverse model, the statistical test, the clustering algorithm and the membership functions we have used to evaluate FuRIA.

We chose to use sLORETA (standardized Low Resolution Electromagnetic TomogrAphy) as the inverse-model due to its good localization properties [9] and the recent success we had using this method in an inverse model based BCI [7]. Using sLORETA, computing the activity in a fuzzy ROI can be done using a simple matrix product:

$$
\gamma_{\Omega_{f}}=m^{T} R_{\Omega_{f}} m \quad \text { with } \quad R_{\Omega_{f}}=\sum_{v=1}^{N_{v}} \mu(v) R_{v}
$$

Here, $m$ is the vector of instantaneous measurements for each one of the $N_{e}$ electrodes used, and $R_{v}$ a $N_{e} * N_{e}$ matrix such that $\gamma_{v}=m^{T} R_{v} m$ [7]. Therefore, computing the activity in a fuzzy ROI is very fast as $R_{\Omega_{f}}$ can be computed offline. To use sLORETA we worked with a head model containing 2394 voxels which is available in the LORETAKey software [10].

Concerning the statistical analysis, we used a permutation t-test with $\alpha=0.01$ [11]. Indeed, this method is nonparametric, enables the computation of the true type I error and is initially designed to study brain voxel activation.

As a clustering algorithm, we employed the Mean Shift algorithm proposed by Comaniciu and Meer [12]. Actually, this nonparametric method can automatically identify any number of arbitrarily shaped clusters. Moreover, this method was recently successfully used for the segmentation of brain MRI, which is a closely related problem [13]. We set the value of the smoothing parameter $H$ to 2 .

During the fuzzification phase, we had to choose the most suitable fuzzy membership functions. Due to the low resolution property of sLORETA, it appears that significant voxels with a relatively high probability of type I error are much more numerous than significant voxels with a relatively low probability of error. Consequently, for each ROI $\Omega$, we chose a function $\mu_{\Omega}$ that assigns a degree of membership that decreases exponentially with the statistic of the voxel:

$$
\begin{gathered}
\mu_{\Omega}(v)= \begin{cases}e^{-\frac{1}{2}\left(\frac{p_{\max }-p_{v}}{\sigma_{\max }}\right)^{2}} & v \in \Omega \\
0 & v \notin \Omega\end{cases} \\
\text { with } \sigma_{\max }=1 /\left(N_{\Omega s} \sum_{p_{v}>0}\left(p_{\min }-p_{v}\right)^{2}\right)
\end{gathered}
$$

$N_{\Omega s}$ is the number of voxels in the ROI $\Omega ; p_{\min }$ and $p_{\max }$ are respectively the minimal and maximal probability of correctly rejecting the null hypothesis $H_{0}$, among the ROI voxels ; and $p_{v}$ is the probability of correctly rejecting $H_{0}$ for voxel $v$. The larger the ROI, the more numerous the voxels with a small $p_{v}$, when using sLORETA. This was taken into account by putting $N_{\Omega s}$ in the $\sigma_{\max }$ denominator.

\section{EVAluATion}

This Section reports a first evaluation of the FuRIA algorithm. First, the EEG data used is presented, then the results are reported and discussed.

\section{A. EEG data set}

To evaluate FuRIA we worked on the EEG data set IV of the BCI competition 2003 [14], provided by the Berlin group [15]. These data contain EEG signals recorded while a subject was performing self-paced left and right finger tapping tasks. EEG signals were sampled at $100 \mathrm{~Hz}$, recorded using 28 electrodes and comprised the $500 \mathrm{~ms}$ before the actual movement. 314 trials were available for training and 100 for testing. The goal of the competitors was to identify, for each trial, the hand used. This results in a binary classification problem with "left" and "right" as classes.

\section{B. Results of the feature extraction with FuRIA}

We trained the FuRIA algorithm on the provided training set. We considered the frequencies between $3 \mathrm{~Hz}$ and 45 $\mathrm{Hz}$, with a $1 \mathrm{~Hz}$ step and focused on the time window that comprises the last $250 \mathrm{~ms}$ of each trial. This means FuRIA worked with the average activity over that time period. FuRIA found five relevant fuzzy ROIs leading to five dimensionnal feature vectors. These fuzzy ROIs and their corresponding frequencies are displayed on Figure 1.

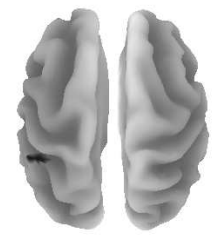

$13 \mathrm{~Hz}$

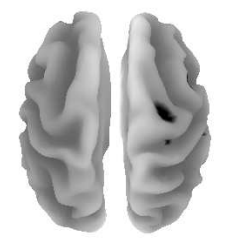

$13 \mathrm{~Hz}$

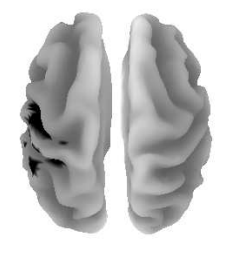

$15-36 \mathrm{~Hz}$

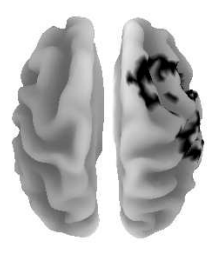

$15-16 \mathrm{~Hz}$

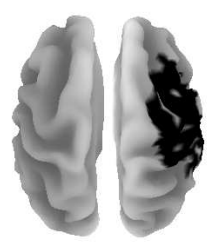

$18-28 \mathrm{~Hz}$
Fig. 1. The fuzzy ROI (in black) and their corresponding frequencies that were automatically obtained by using FuRIA. The darker the voxel color, the higher the voxel degree of membership $\mu(v)$. The brain is inflated and is seen from the top, front up. These pictures were obtained with the LORETAKey software [10].

We can notice that FuRIA found two ROIs which corresponding frequencies are in the $\alpha$ rhythm $(13 \mathrm{~Hz})$ and three 
ROIs with frequencies in the $\beta$ rhythm $(15-16 \mathrm{~Hz}, 15-36 \mathrm{~Hz}$, 18-28 Hz). Moreover, the ROIs found stood in the left and right motor cortex, which is consistent with the knowledge about movement intention [16].

\section{Classification}

To classify the features extracted using FuRIA, we used a linear Support Vector Machine (SVM). Actually, this classifier is one of the most popular and efficient classifier in BCI research [2]. This classifier was classically trained on the training data and then used to classify the features of the test set. The range of accuracy obtained after 100 repetition of training/testing is displayed in Table I and compared with two other methods: the one of the winner of the competition as a reference and a method using inverse models we proposed previously. The results using FuRIA without the fuzzification step (step 5 in Section II-A) are also displayed for comparison.

TABLE I

ClassificATION ACCURACY FOR THE TEST SET (\%)

\begin{tabular}{|c||c|}
\hline Method & Test Set \\
\hline BCI competition 2003 Winner [14] & 84 \\
\hline Previous work using inverse models [7] & 83 \\
\hline FuRIA without fuzzification & $80-82$ \\
\hline FuRIA with fuzzification & $\mathbf{8 5 - 8 6}$ \\
\hline
\end{tabular}

We can notice that the method which uses FuRIA with fuzzification reached the highest score with an accuracy ranging from $85 \%$ to $86 \%$. These results suggest that the features extracted are very discriminative as they are very few but still enable good classification results. We can also notice that when using FuRIA without fuzzification, the results are significantly lower. This highlights the interest of using efficiently the information contained in any significant voxel thanks to the concept of fuzzy ROI. Finally, it is worth noting that in the methods proposed in [7] and [14], data driven spatial filters were used to increase performances. This suggests that combining FuRIA with efficient spatial filters such as the ones described in [7] could lead to better results.

\section{CONCLUSION AND FUTURE WORK}

In this paper, we have proposed a trainable feature extraction algorithm for Brain-Computer Interfaces (BCIs) called FuRIA (Fuzzy Region of Interest Activity). This algorithm is based on inverse models and makes use of the novel concept of fuzzy Region Of Interest (ROI). FuRIA can automatically find relevant fuzzy ROIs for discrimination as well as the frequency bands in which they react. The activity in these ROIs could then be used as features for any classifier. FuRIA has been evaluated on data set IV of the BCI competition 2003 using a Support Vector Machine as classifier. Results are promising as this method reached a higher accuracy on the test set than the winner of this competition.

Future work will be dedicated to the evaluation of FuRIA on different data sets corresponding to other mental tasks.
This will include the evaluation of FuRIA for multiclass BCIs. Indeed, adaptations seem necessary to tackle such BCIs as t-tests would not be suitable for problems with more than two classes. ANOVA tests could solve such an issue. It would be also interesting to study the influence of the frequential and spatial sampling on the performance.

\section{ACKNOWLEDGMENTS}

The authors would like to thank Pr. Oscar Yanez-Suarez, for its help concerning the Mean Shift algorithm, as well as Morgane Rosendale, Stéphanie Gerbaud, Yann Renard and Marco Congedo for their contribution on a preliminary draft of the manuscript.

\section{REFERENCES}

[1] J. R. Wolpaw, N. Birbaumer, D. J. McFarland, G. Pfurtscheller, and T. M. Vaughan, "Brain-computer interfaces for communication and control," Clinical Neurophysiology, vol. 113, no. 6, pp. 767-791, 2002.

[2] F. Lotte, M. Congedo, A. Lécuyer, F. Lamarche, and B. Arnaldi, "A review of classification algorithms for eeg-based brain-computer interfaces," Journal of Neural Engineering, 2007, to appear.

[3] D. J. McFarland, C. W. Anderson, K.-R. Muller, A. Schlogl, and D. J. Krusienski, "Bci meeting 2005-workshop on bci signal processing: feature extraction and translation," IEEE Transactions on Neural Systems and Rehabilitation Engineering, vol. 14, no. 2, pp. 135 138, 2006.

[4] L. Qin, L. Ding, and B. He, "Motor imagery classification by means of source analysis for brain computer interface applications," Journal of Neural Engineering, vol. 1, no. 3, pp. 135-141, 2004.

[5] R. Grave de Peralta Menendez, S. Gonzalez Andino, L. Perez, P. Ferrez, and J. Millán, "Non-invasive estimation of local field potentials for neuroprosthesis control," Cognitive Processing, Special Issue on Motor Planning in Humans and Neuroprosthesis Control, 2005.

[6] M. G. Wentrup, K. Gramann, E. Wascher, and M. Buss, "Eeg source localization for brain-computer-interfaces," in 2nd International IEEE EMBS Conference on Neural Engineering, 2005, pp. 128-131.

[7] M. Congedo, F. Lotte, and A. Lécuyer, "Classsification of movement intention by spatially filtered electromagnetic inverse solutions,' Physics in Medicine and Biology, vol. 51, no. 8, pp. 1971-1989, 2006.

[8] L. A. Zadeh, "Fuzzy sets," Fuzzy sets, fuzzy logic, and fuzzy systems: selected papers by Lotfi A. Zadeh, pp. 19-34, 1996.

[9] R. D. Pascual-Marqui, "Standardized low resolution brain electromagnetic tomography (sloreta): technical details," Methods and Findings in Experimental and Clinical Pharmacology, vol. 24D, pp. 5-12, 2002.

[10] — " "http://www.unizh.ch/keyinst/newloreta/loreta01.htm."

[11] A. P. Holmes, R. C. Blair, J. D. Watson, and I. Ford, "Nonparametric analysis of statistic images from functional mapping experiments," Journal of Cerebral Blood Flow and Metabolism, vol. 16, pp. 7-22, 1996.

[12] D. Comaniciu and P. Meer, "Mean shift - a robust approach toward feature space analysis," IEEE Transactions on Pattern Analysis and Machine Intelligence, vol. 24, no. 5, pp. 603-619, 2002.

[13] J. Jimenez-Alaniz, M. Pohl-Alfaro, V. Medina-Banuelos, and O. Yanez-Suarez, "Segmenting brain mri using adaptive mean shift," in 28th International IEEE EMBS Annual Conference, 2006, pp. 3114 $-3117$.

[14] Y. Wang, Z. Zhang, Y. Li, X. Gao, S. Gao, and F. Yang, "Bci competition 2003-data set iv: an algorithm based on cssd and fda for classifying single-trial eeg," IEEE Transactions on Biomedical Engeneering, vol. 51, no. 6, pp. 1081-1086, 2004.

[15] B. Blankertz, G. Curio, and K. R. Müller, "Classifying single trial eeg: Towards brain computer interfacing," Advances in Neural Information Processing Systems (NIPS 01), vol. 14, pp. 157-164, 2002.

[16] G. Pfurtscheller, "Eeg event-related desynchronization (erd) and eventrelated synchronization (ers)," Electroencephalography: Basic Principles, Clinical Applications and Related Fields, 4th ed., pp. 958,967, 1999. 